Danyane Simão Gomes,

Alanna Simão G. Saturnino, Danielle de Freitas Gonçalves,

Carlos Bandeira de M. Monteiro e Maria Georgina M. Tonello

\title{
USO DA REALIDADE VIRTUAL COMO ESTRATÉGIA DE REABILITAÇÃO E LAZER PARA PESSOAS COM LESÃO DA MEDULA ESPINAL: UMA REVISÃO INTEGRATIVA
}

Recebido em: $21 / 11 / 2018$

Aceito em: 17/05/2019

\author{
Danyane Simão Gomes ${ }^{1}$ \\ Alanna Simão Gomes Saturnino ${ }^{2}$ \\ Danielle de Freitas Gonçalves ${ }^{3}$ \\ Universidade de Franca (UNIFRAN) \\ Franca - SP - Brasil \\ Carlos Bandeira de Mello Monteiro ${ }^{4}$ \\ Universidade de São Paulo (USP) \\ São Paulo - SP - Brasil \\ Maria Georgina Marques Tonello ${ }^{5}$ \\ Universidade de Franca (UNIFRAN) \\ Franca - SP - Brasil
}

RESUMO: Intervenções com a Realidade Virtual (RV) tem sido descritas como alternativas para o tratamento de pessoas com lesão da medula espinal (LME). O presente estudo buscou oferecer uma visão geral das intervenções que utilizaram a RV na reabilitação e como estratégia de lazer destes indivíduos. Foi realizada uma revisão sistemática, nas bases de dados PubMed (Medline), Biblioteca Virtual em Saúde (BVS), Scielo e Lilacs nos últimos cinco anos. Um total de 13 artigos foram identificados. A RV foi utilizada para diversos objetivos como: verificar a melhora da função dos membros superiores; associar com estímulos sensoriais durante a marcha; identificar os efeitos da caminhada virtual sobre o grau de dor. Assim, percebe-se que a RV pode ser usada para diversas finalidades, além de fornecer um meio mais envolvente de

\footnotetext{
${ }^{1}$ Doutoranda em Promoção de Saúde pela Universidade de Franca (UNIFRAN) e Mestre em Fisioterapia pelo Centro Universitário do Triângulo (UNITRI). Docente do Centro Universitário de Patos de Minas (UNIPAM).

${ }^{2}$ Especialista em Clínica Médica do Hospital Regional de Sobradinho/SESDF. Graduação em Medicina pelo Centro Universitário de Patos de Minas (UNIPAM).

${ }^{3}$ Doutoranda em Promoção de Saúde pela Universidade de Franca (UNIFRAN) e Mestre em Promoção de Saúde (UNIFRAN).

${ }^{4}$ Doutor em Ciências na área de Neurologia pela Faculdade de Medicina da Universidade de São Paulo (USP/FMUSP*). Docente do curso de Educação Física e Saúde da Escola de Artes, Ciências e Humanidades da Universidade de São Paulo (EACH/USP).

${ }^{5}$ Doutora em Educação Especial pela Universidade Federal de São Carlos (UFSCar). Docente do Programa de Mestrado e Doutorado em Promoção de Saúde (UNIFRAN). Coordenadora do Grupo de Pesquisa "Promoção de saúde para pessoas com deficiências lazer e estilo de vida ativo".
} 
Danyane Simão Gomes,

Alanna Simão G. Saturnino, Danielle de Freitas Gonçalves,

Carlos Bandeira de M. Monteiro e Maria Georgina M. Tonello

tratamento para pessoas com LME, adicionando uma dimensão de prazer e um momento para o lazer.

PALAVRAS CHAVE: Realidade Virtual. Reabilitação. Traumatismos da Medula Espinal.

\section{USE OF VIRTUAL REALITY AS A REHABILITATION AND LEISURE STRATEGY FOR PEOPLE WITH SPINAL CORD INJURY: A INTEGRATIVE REVIEW}

ABSTRACT: Interventions with Virtual Reality (VR) have been described as alternatives for the treatment of people with spinal cord injury (LME). The present study sought to provide an overview of the interventions that used VR in the rehabilitation of these individuals. A systematic review was performed in the PubMed (Medline), Virtual Health Library (VHL), Scielo and Lilacs databases in the last 5 years. A total of 13 articles have been identified. VR was used for several purposes such as: to verify the improvement of upper limb function; associated with sensory stimuli during gait; to identify the effects of virtual walking on the degree of pain. Thus, it can be seen that RV can be used for different purposes, as well as providing a more involving means of treatment for people with LME, adding a dimension of pleasure and a moment for leisure.

KEYWORDS: Virtual Reality. Rehabilitation. Spinal Cord Injuries.

\section{Introdução}

A reabilitação de pessoas com lesão da medula espinal (LME) possui uma abordagem interdisciplinar, incluindo médicos, fisioterapeutas, enfermeiros, psicólogos, nutricionistas, dentre outros. Ela irá gerar efeitos significativos em longo prazo, auxiliando-os a recuperar suas funções, evitar complicações secundárias, compreender a extensão de suas lesões, e auxiliá-los a lidar com a perda de independência (AHUJA et al., 2017).

O avanço tecnológico pode oferecer novos caminhos para a reabilitação e várias intervenções terapêuticas tem sido apresentadas. A Realidade Virtual (RV) faz parte deste novo cenário, demonstrando ser uma alternativa de tratamento que tem se tornado cada vez mais popular e não é somente capaz de fornecer um feedback sobre o 
Danyane Simão Gomes,

Alanna Simão G. Saturnino, Danielle de Freitas Gonçalves,

Carlos Bandeira de M. Monteiro e Maria Georgina M. Tonello

seu desempenho motor, mas também, por meio de ambientes virtuais, gerar maior engajamento e motivação durante a reabilitação dos indivíduos.

Esse processo de recuperação e tratamento das pessoas com LME muitas vezes mostra-se prologando, repetitivo, caro e monótono e como forma de estimular estes indivíduos, a RV tem sido bastante recomendada. Neste sentido, observa-se que os jogos interativos/virtuais tem sido utilizados na reabilitação a fim de gerar entusiasmo e motivação durante as sessões, além de curiosidade, exploração, competição e interação social. Ela torna esse processo mais dinâmico, ativo, agradável, podendo ter influência positiva sobre a qualidade de vida destas pessoas, pois acrescenta prazer durante a execução da tarefa, oferecendo também oportunidade para socialização e lazer (PRASAD et al., 2018).

Existem diversos equipamento de jogos virtuais, tais como o Nintendo Wii e o X-Box 360. Estes são exemplos de instrumentos de baixo custo e que podem ser utilizados em longo prazo de forma complementar à reabilitação tradicional ou até mesmo nos domicílios de pessoas com LME, podendo assim, atender também às necessidades de lazer e interação social.

Segundo Serra; Fava e Tonello (2018), além das melhorias terapêuticas tradicionais, a RV apresenta diversos benefícios sociais para pessoas com deficiência, uma vez que tem se tornado uma atividade de lazer para as mesmas. Dentre tais benefícios, destaca-se a interação e prevenção do isolamento social, além de atuar na promoção da saúde física dessa população, por ser considerada uma maneira efetiva de praticar exercícios físicos.

Ademais, a realização de atividades de lazer por meio da RV mostra-se eficaz para a restauração psicossocial de pessoas com LME, atuando na redução do estresse e 
Danyane Simão Gomes,

Alanna Simão G. Saturnino, Danielle de Freitas Gonçalves,

Carlos Bandeira de M. Monteiro e Maria Georgina M. Tonello

alcance de habilidades de enfrentamento, visto que, a prática fornece oportunidades de vivenciar situações que poderiam se tornar inalcançáveis devido às suas limitações motoras (SERRA; FAVA E TONELLO, 2018).

Nota-se, desta forma, que a RV pode ser utilizada para diversas finalidades, variando desde à reabilitação até ao lazer. De acordo com Zimmerli et al. (2013), o uso da "caminhada virtual interativa" pode oferecer uma ilusão de marcha normal para pessoas com LME e, isso pode melhorar suas funções motoras. Já Kumru et al. (2013) afirmam que o uso desta técnica pode também diminuir a dor neuropática gerada após este tipo de lesão.

Assim, considerando os benefícios que a RV tem proporcionado aos indivíduos com LME, este estudo se justifica, pois se acredita que intervenções de reabilitação baseadas em RV tem sido um recurso altamente utilizado para melhorar a função, a deambulação e o equilíbrio de indivíduos com lesões neurológicas, conforme relatado por Wall et al. (2015). Além disto, ela tem demonstrado ser uma importante ferramenta para estimular o engajamento e a motivação dos indivíduos durante o processo de reabilitação, podendo gerar um momento de prazer e diversão para eles.

Neste sentido, o objetivo deste artigo foi identificar, por meio de uma revisão sistemática da literatura, como a RV tem sido utilizada no tratamento de pessoas com LME, suas diversas abordagens e os resultados encontrados.

\section{Material e Métodos}

Tratou-se de uma revisão sistemática da literatura, a qual se iniciou com a definição da pergunta norteadora ("Como a RV tem sido utilizada na reabilitação de pessoas com LME?”). Posteriormente foi realizado um levantamento bibliográfico nas 
Danyane Simão Gomes,

Alanna Simão G. Saturnino, Danielle de Freitas Gonçalves,

Carlos Bandeira de M. Monteiro e Maria Georgina M. Tonello

bases de dados eletrônicas Pubmed (Medline), Portal de Busca da Biblioteca Virtual de Saúde (BVS), Scielo e Lilacs.

Foram utilizados os seguintes descritores: "spinal cord injury", "virtual reality", "virtual feedback", "visual feedback", "game-based virtual reality”, e as combinações dessas palavras-chave: "spinal cord injury AND virtual reality"; "spinal cord injury AND virtual feedback"; "spinal cord injury AND visual feedback" e "spinal cord injury AND game-based virtual reality".

Foram incluídos artigos publicados entre janeiro de 2014 a janeiro de 2019, pelo idioma inglês, os quais utilizaram a RV em pessoas com LME. Foram excluídos trabalhos cuja amostra tenha sido exclusivamente composta por indivíduos com lesão incompleta da medula, amostra composta por crianças, idosos ou animais, trabalhos de revisão bibliográfica e estudos de caso.

Por meio da busca realizada nas bases de dados citadas anteriormente, foram encontrados 147 artigos. Após a leitura dos resumos, foram excluídos os artigos repetidos, aplicado os critérios de inclusão e exclusão e finalmente, foram selecionados 13 artigos que atendiam aos objetivos do presente estudo (Figura 1).

Figura 1: Fluxograma de busca e seleção dos artigos utilizados no estudo.

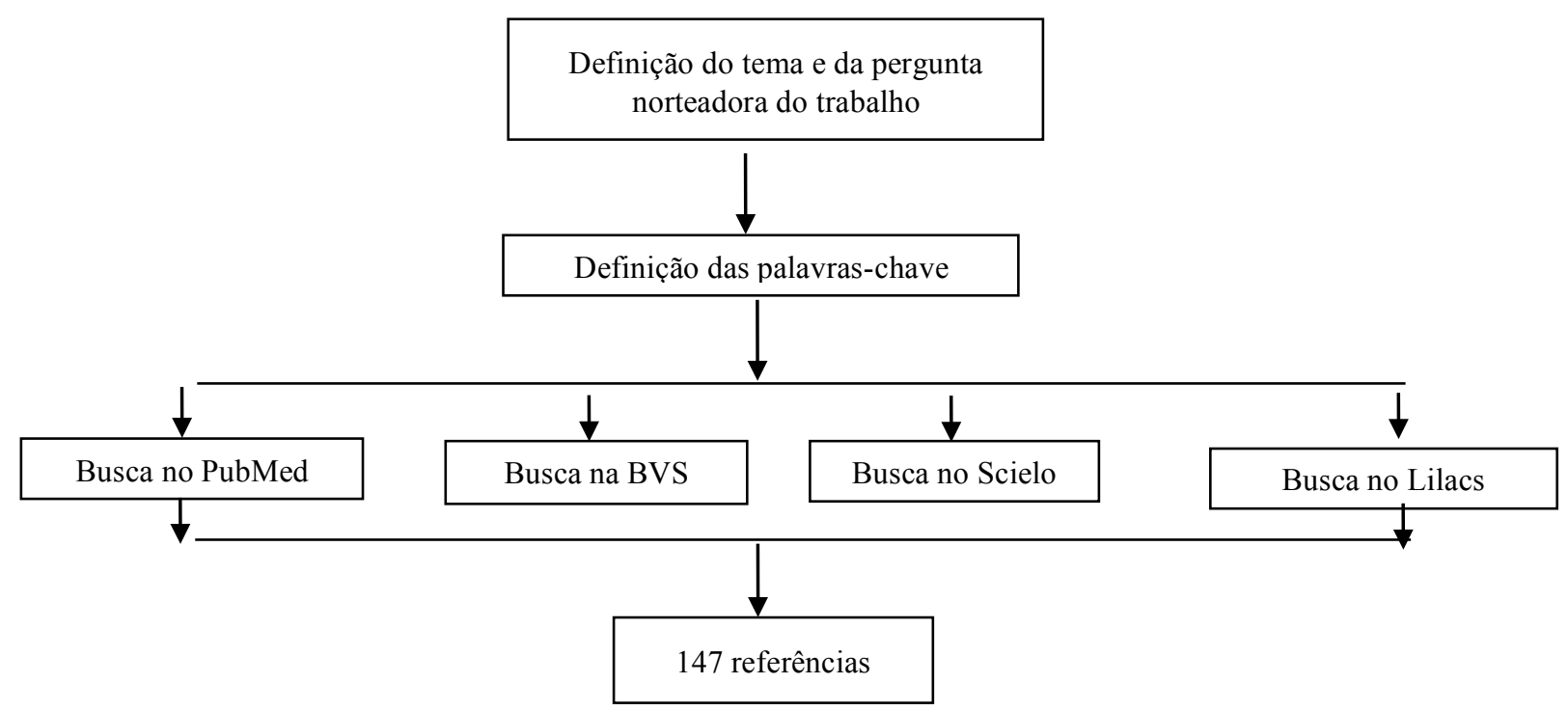


Danyane Simão Gomes,

Alanna Simão G. Saturnino, Danielle de Freitas Gonçalves,

Carlos Bandeira de M. Monteiro e Maria Georgina M. Tonello

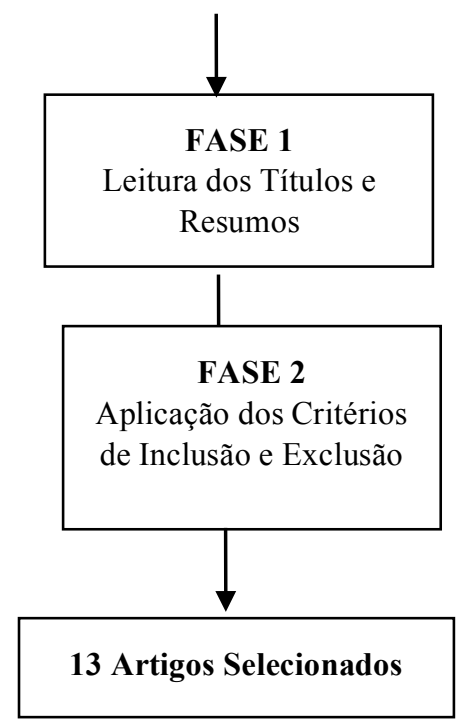

\section{Resultados e Discussão}

Foram identificados 147 estudos que relacionaram a RV e a reabilitação de pessoas com LME. No entanto, a partir da aplicação dos critérios previamente definidos, apenas 13 fizeram parte do escopo desta revisão. Dentre estes, cinco avaliaram o efeito da RV sobre a função dos membros superiores (MMSS); dois associaram o uso da estimulação sensorial/tátil durante a simulação da marcha em um ambiente virtual; dois verificaram os efeitos da caminhada virtual sobre o grau de dor; dois testaram os efeitos para treinar os usuários em cadeira de rodas; um identificou a localização da ativação cortical durante a prática da caminhada virtual; e um verificou o efeito da caminhada virtual para auxiliar no uso do exoesqueleto (Tabela 1). 
Danyane Simão Gomes,

Alanna Simão G. Saturnino, Danielle de Freitas Gonçalves,

Carlos Bandeira de M. Monteiro e Maria Georgina M. Tonello

Tabela 1: Intervenções de RV para a reabilitação de pessoas com LME.

\begin{tabular}{|c|c|c|c|}
\hline Autores/ Ano & $\begin{array}{c}\text { Amostra /Parâmetros } \\
\text { avaliados }\end{array}$ & $\begin{array}{c}\text { Procedimentos de Realidade } \\
\text { Virtual }\end{array}$ & Resultados \\
\hline $\begin{array}{l}\text { PRASAD, S. et } \\
\text { al. (2018). }\end{array}$ & $\begin{array}{c}\mathrm{n}=20 \text { (GE: } 11 \text { e GC: } \\
\text { 9). } \\
\text { Ensaio piloto } \\
\text { randomizado. } \\
\text { CUE; BBT, SCIM; } \\
\text { Avaliação da } \\
\text { satisfação. }\end{array}$ & $\begin{array}{l}\text { GE: } 12 \text { sessões de intervenção } \\
(\mathrm{NWii}+\mathrm{TC}) \\
\text { GC: } 12 \text { sessões de TC }\end{array}$ & $\begin{array}{l}\text { A análise entre grupos não } \\
\text { mostrou melhora significativa no } \\
\text { questionário CUE, escores do } \\
\text { BBT, SCIM e WHOQOL-BREF. } \\
\text { No entanto, os escores médios no } \\
\text { GE foram maiores do que aqueles } \\
\text { no grupo de controle. Quanto à } \\
\text { avaliação da satisfação com a } \\
\text { terapia baseada no Wii, os } \\
\text { pacientes estavam muito } \\
\text { satisfeitos. }\end{array}$ \\
\hline $\begin{array}{l}\text { KHURANA, M.; } \\
\text { WALIA, S.; } \\
\text { NOOHU, M.M. } \\
\text { (2017). }\end{array}$ & $\begin{array}{l}\mathrm{n}=30 \text { indivíduos } \\
\text { (Grupo A e B). } \\
\text { mFRT; Teste de } \\
\text { autonomia funcional; } \\
\text { SCIM-III. }\end{array}$ & $\begin{array}{l}\text { Grupo A: treinamento com jogos } \\
\text { de RV (Sony PlayStation } 2 \text { e } \\
\text { EyeToy) } \\
\text { Grupo B: treinamento específico } \\
\text { para tarefas. } \\
\text { (20 sessões). }\end{array}$ & $\begin{array}{l}\text { Grupo A apresentou melhoria no } \\
\text { equilíbrio e desempenho } \\
\text { funcional. }\end{array}$ \\
\hline $\begin{array}{l}\text { POZEG, P. et al. } \\
\text { (2017). }\end{array}$ & $\begin{array}{c}\mathrm{n}=40 \text { (GE: } 20 \\
\text { indivíduos com LME e } \\
\text { GC: } 20 \text { saudáveis). } \\
\text { IPV: Questionário; } \\
\text { IGC: Questionário; } \\
\text { EVA; Escala de } \\
\text { Despersonalização de } \\
\text { Cambridge. }\end{array}$ & $\begin{array}{l}\text { IPV: pernas falsas foram } \\
\text { colocadas em outra cadeira; uma } \\
\text { câmera foi montada acima, } \\
\text { correspondendo ao ponto de } \\
\text { vista em primeira pessoa. A } \\
\text { gravação de vídeo em tempo real } \\
\text { das pernas virtuais aparecia } \\
\text { como sobrepostas às pernas } \\
\text { físicas dos participantes, } \\
\text { enquanto o pesquisador gerava } \\
\text { estímulo tátil. IGC: simular ao } \\
\text { anterior, porém a câmera foi } \\
\text { posicionada atrás, filmando as } \\
\text { costas do participante (1 sessão). }\end{array}$ & $\begin{array}{l}\text { Pacientes com LME são menos } \\
\text { sensíveis a estímulos } \\
\text { multissensoriais de indução de } \\
\text { posse de uma perna ilusória. } \\
\text { Ilusão da perna virtual e o corpo } \\
\text { global foram ambos associados } \\
\text { com analgesia leve. }\end{array}$ \\
\hline
\end{tabular}


Danyane Simão Gomes,

Alanna Simão G. Saturnino, Danielle de Freitas Gonçalves,

Carlos Bandeira de M. Monteiro e Maria Georgina M. Tonello

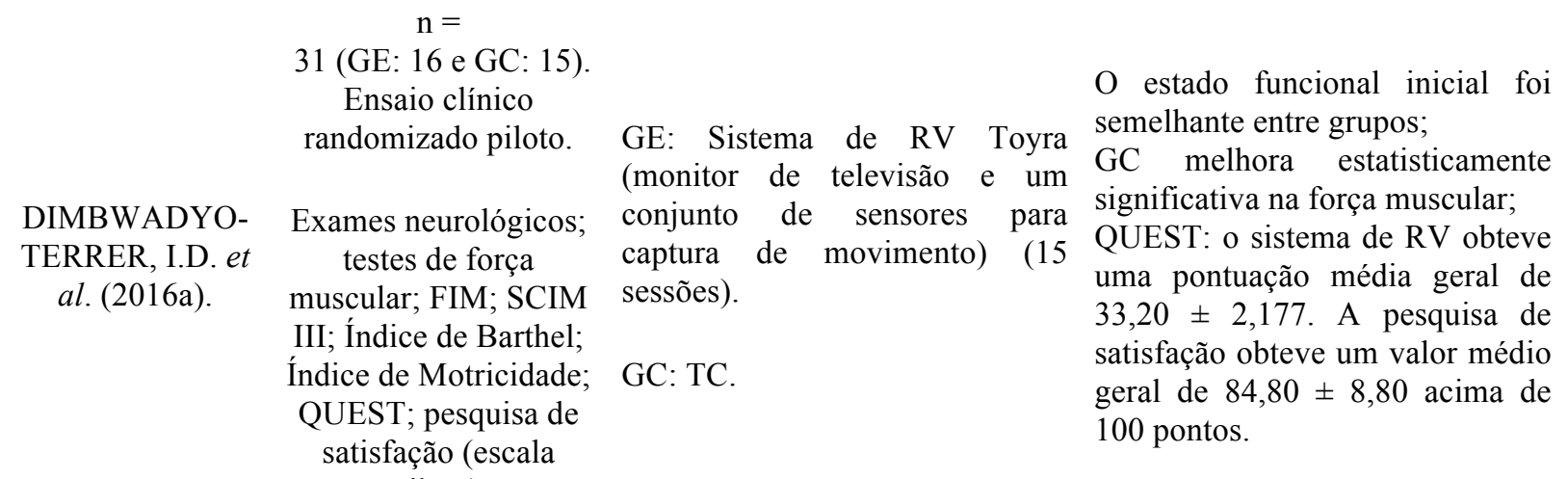

Likert).

\begin{tabular}{|c|c|c|c|}
\hline $\begin{array}{l}\text { DIMBWADYO- } \\
\text { TERRER, I. et al. } \\
\text { (2016b). }\end{array}$ & $\begin{array}{c}\mathrm{n}=9 \text { (GE: } 6 \text { e GC: } 3) . \\
\text { Estudo-piloto. } \\
\text { Força muscular; Índice } \\
\text { de Barthel; SCIM; } \\
\text { NHPT; JHFT. }\end{array}$ & $\begin{array}{l}\text { GE: O usuário era capaz de ver } \\
\text { sua mão virtual na cena, assim } \\
\text { como objetos virtuais exibidos } \\
\text { em um monitor. Três tarefas } \\
\text { virtuais foram executadas para } \\
\text { melhorar os movimentos de } \\
\text { alcance funcional do paciente. } \\
\text { GC: TC. } \\
\text { (4 sessões). }\end{array}$ & $\begin{array}{l}\text { O estado funcional inicial foi } \\
\text { semelhante entre os grupos. GE } \\
\text { apresentou maiores escores para } \\
\text { força muscular. A escala SCIM } \\
\text { mostrou indicações favoráveis na } \\
\text { avaliação final tanto no em } \\
\text { ambos os grupos. Não foram } \\
\text { obtidas diferenças } \\
\text { estatisticamente significantes em } \\
\text { relação à destreza, coordenação e } \\
\text { avaliação dos movimentos finos } \\
\text { dos dedos. }\end{array}$ \\
\hline
\end{tabular}


Danyane Simão Gomes,
Alanna Simão G. Saturnino, Danielle de Freitas Gonçalves,

Carlos Bandeira de M. Monteiro e Maria Georgina M. Tonello

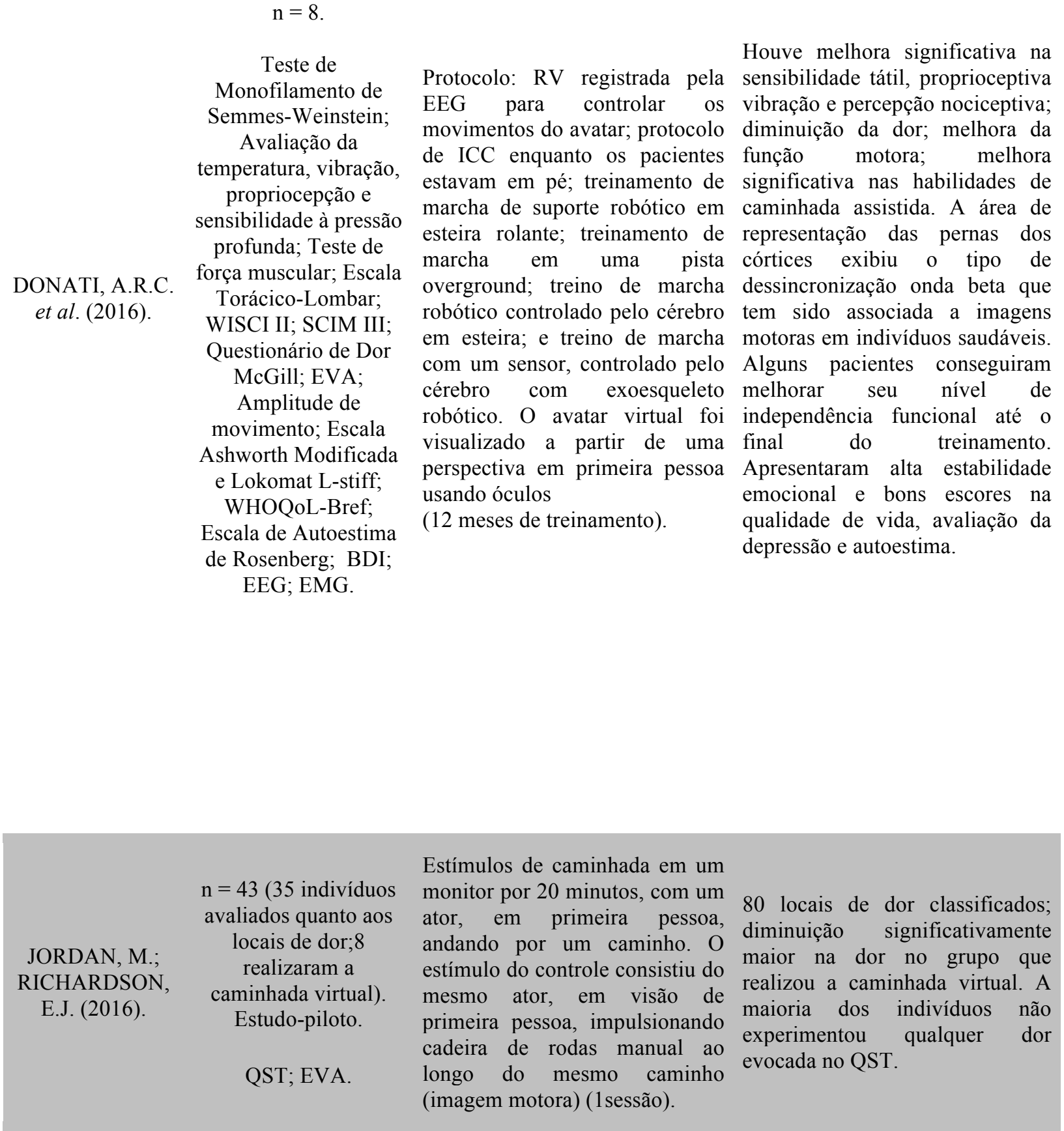


Danyane Simão Gomes,
Alanna Simão G. Saturnino, Danielle de Freitas Gonçalves,

Carlos Bandeira de M. Monteiro e Maria Georgina M. Tonello
NUNNERLEY, J. et al. (2017). $\mathrm{n}=12$ (5 usuários experientes em cadeira de rodas com diagnóstico de LME e 7 médicos participantes).

Grupos focais e entrevistas individuais.
Os participantes testaram o instrutor de cadeira de rodas: os usuários de cadeiras de rodas permaneceram em suas próprias cadeiras e os médicos participantes usaram uma cadeira de escritório com altura ajustável, sentado em uma mesa, na frente de um computador. Os óculos foram configurados com a cadeira de rodas e o controle preso à mesa. Os participantes passaram 20 a 30 minutos testando o sistema (1 sessão).
O sistema simulou realisticamente a experiência de dirigir uma cadeira de rodas elétrica. Algumas áreas onde o sistema necessitava de realismo também foram destacadas. Os usuários experientes em cadeira de rodas lembraram sentimentos de ansiedade e futuro desconhecido depois de experimentar a LME e alguns explicaram como o sistema de RV poderia ajudar. Um dos usuários sentiu que o sistema RV seria uma maneira "divertida" de aprender habilidades em cadeiras de rodas e os clínicos identificaram o sistema como forma de engajamento precoce.

\begin{tabular}{|c|c|c|c|}
\hline $\begin{array}{l}\text { ROOSINK, M. et } \\
\text { al. (2016). }\end{array}$ & $\begin{array}{l}\mathrm{n}=9 . \\
\text { Conjunto de dados da } \\
\text { dor básica; HADS; } \\
\text { KVIQ-10; } \\
\text { Classificação da } \\
\text { vivacidade da imagem } \\
\text { motora percebida e } \\
\text { esforço de imagens } \\
\text { motoras; EVA. }\end{array}$ & $\begin{array}{l}\text { Sessões de caminhada virtual } \\
\text { interativa, equipados com } 2 \\
\text { braçadeiras reguláveis contendo } \\
\text { um sensor de movimento inercial } \\
\text { e usando óculos 3d. Foram } \\
\text { instruídos a mentalizar imagens } \\
\text { da marcha (imagem motora) a } \\
\text { uma velocidade confortável, } \\
\text { enquanto iam balançando seus } \\
\text { braços de acordo com a } \\
\text { velocidade imaginada da marcha } \\
\text { e enquanto se concentravam nas } \\
\text { sensações corporais produzidas } \\
\text { por imagens de marcha (2 } \\
\text { sessões). }\end{array}$ & $\begin{array}{l}7 \text { participantes relataram dor } \\
\text { neuropática e outros tipos de dor } \\
\text { também foram relatadas } \\
\text { (musculoesquelético } n=5 \text {, } \\
\text { visceral } n=1 \text {, cefaleia } \mathrm{n}=1 \text { ). A } \\
\text { capacidade geral das imagens } \\
\text { motoras foi muito variável entre } \\
\text { os participantes, variando de } \\
\text { muito pobre a muito bom. Houve } \\
\text { efeitos significativos do feedback } \\
\text { visual e direção de velocidades } \\
\text { motoras e de Feedback visual } \\
\text { para vivacidade motora das } \\
\text { imagens e esforço. A presença do } \\
\text { avatar foi associado com melhora } \\
\text { da vivacidade motora e } \\
\text { velocidade de imagens e com } \\
\text { esforço imagético motor inferior. } \\
\text { Boa interação com o avatar e a } \\
\text { cena virtual. }\end{array}$ \\
\hline
\end{tabular}


Danyane Simão Gomes,

Alanna Simão G. Saturnino, Danielle de Freitas Gonçalves,

Carlos Bandeira de M. Monteiro e Maria Georgina M. Tonello

$\mathrm{n}=8$.

Teste de pseudopropriocepção; teste de

SHOKUR, S. et al. (2016) tarefa transversal

congruente; teste de

tarefa envolvendo a

simulação de texturas de piso.
Uso de camisa tátil e um óculos A exibição tátil induziu a ilusão de realidade virtual na cabeça, realista de andar nos três tipos no qual um avatar humano 3D diferentes de superfícies em seis era projetado e podia ficar de pé pacientes. Além disso, os e andar em três diferentes tipos pacientes experimentaram os de superfícies (grama, areia e rua movimentos dos MMII virtuais pavimentada), enquanto feedback tátil era reproduzido no antebraçon (15 sessões). durante a fase de balanço ou a sensação do pé rolando no chão enquanto caminhava.
$\mathrm{n}=18(\mathrm{GE}: 9$ e GC:

9).

WANG, Y. T. et al. (2015).

Teste de competência; teste de retenção/freio

e teste de transferência.
GE: feedback de vídeo imediato

para treinamento de habilidade avançada de cadeira de rodas. GC: feedback fornecido pelos fisioterapeutas )

(2 vezes/semana até o participante dominar as
Não houve diferença significativa entre os grupos no tempo de treinamento necessário. Também não houve diferença significativa entre os dois grupos nas três habilidades de cadeira de rodas. habilidades).

$\mathrm{n}=8$ (GE: 3 LME e

GC: 5 saudáveis).

EICK, J.; RICHARDSON, E.J. (2015).

Estudo-piloto.

Ressonância
Magnética Cerebral;

Avaliação do quão imersivo / realista foi a adaptação a cenas virtuais; Avaliação da capacidade de imaginar que estavam realizando os movimentos dos MMII.
Os estímulos de caminhada Houve ativação significativa ao consistiram em um vídeo de um longo do córtex somatossensorial ator andando por um caminho, bilateral e do lóbulo paracentral, e em visão de primeira pessoa, em menor grau, embora ainda impulsionando uma cadeira de presente, das áreas motoras rodas manual pelo caminho. Os mediais. GC apresentou participantes foram instruídos a significativa ativação nos córtex imaginar que eles próprios frontal e pré-motor. Os estímulos estavam realizando os visuais ilusórios foram movimentos (imagem motora) (1 experimentados como mais sessão). imersivos no GC do que no GE. 
Danyane Simão Gomes,

Alanna Simão G. Saturnino, Danielle de Freitas Gonçalves,

Carlos Bandeira de M. Monteiro e Maria Georgina M. Tonello

\begin{tabular}{|c|c|c|c|}
\hline $\begin{array}{l}\text { TRINCADO- } \\
\text { ALONSO, F. et } \\
\text { al. (2014). }\end{array}$ & $\begin{array}{c}\mathrm{n}=15 \\
\text { SCIM II; Escala de } \\
\text { índice motor do } \\
\text { MMSS; FIM. }\end{array}$ & $\begin{array}{l}\text { Cinco sensores de movimento } \\
\text { (tronco, costas, cabeça, } \\
\text { antebraço e mão) foram usados } \\
\text { para capturar movimentos dos } \\
\text { MMSS dominantes, conectado } \\
\text { via Bluetooth a um computador, } \\
\text { que foi responsável para a } \\
\text { sincronização, coleta de dados e } \\
\text { transmissão. Cada sujeito } \\
\text { recebeu uma explicação sobre } \\
\text { como realizar a atividade, que } \\
\text { consistia em atingir os diferentes } \\
\text { objetivos que apareciam } \\
\text { sequencialmente na tela (1 } \\
\text { sessão). }\end{array}$ & $\begin{array}{l}\text { Correlações positivas fortes: } \\
\text { SCIM II e flexão do ombro; } \\
\text { SCIM e flexão completa do } \\
\text { ombro; índice motor do MMSS e } \\
\text { flexão do ombro; amplitude } \\
\text { articular e o SCIM; SCIM e } \\
\text { índice motor do MMSS. } \\
\text { Correlações positivas moderadas: } \\
\text { SCIM e abdução do ombro; } \\
\text { SCIM e abdução completa do } \\
\text { ombro; SCIM e desvio ulnar; } \\
\text { índice motor do MMSS e } \\
\text { abdução do ombro; FIM e } \\
\text { abdução do ombro; FIM e flexão } \\
\text { completa do ombro; amplitude } \\
\text { articular e FIM. Correlação } \\
\text { negativa moderada: agilidade e } \\
\text { índice motor do MMSS. }\end{array}$ \\
\hline
\end{tabular}

Abreviações - BBT: Teste de caixa e bloco; BDI: Inventário de Depressão de Beck; CUE: Questionário para habilidade funcional da mão-alvo; GC: grupo controle; GE: grupo experimento; EEG: eletroencefalograma; EMG: eletromiografia; EVA: escala analógica visual de dor; FIM: medida de independência funcional; HADS: Escala hospitalar de Ansiedade e Depressão; ICC: interface-cérebrocomputador; IGC: Ilusão global corporal; IPV: Ilusão das pernas virtuais; JHFT: Teste Jebsen Taylor Hand Function; KVIQ-10: Questionário cinestésico e de imagens visuais; LME: lesão da medula espinal; mFRT: Teste de alcance funcional modificado; MMSS: membros superiores; MMII: membros inferiores; NHPT: Teste de nine hole peg; NWii: Nintendo Wii; QST: Teste quantitativo sensorial; QUEST: Avaliação da satisfação do usuário com a tecnologia assistiva 2.0; RV: realidade virtual; SCIM: medida de independência da medula espinal; TC: terapia convencional; WHOQOL-BREF: questionário de avaliação da qualidade de vida da Organização Mundial da Saúde; WISCI: Índice de marcha de lesão da medula espinal.

O presente artigo buscou identificar o uso da RV na reabilitação de pessoas com LME. De acordo com os resultados apresentados, percebe-se que a RV pode ser utilizada de diversas maneiras na reabilitação destes indivíduos, indicando diversos benefícios para o seu uso, tais como o estímulo das funções dos MMSS, treino de cadeiras de rodas ou exoesqueleto, diminuição de dor neuropática e principalmente como um instrumento que proporciona grande satisfação a essas pessoas, portanto, apesar do lazer não ser um descritor utilizado nesse estudo, a maioria dos resultados encontrados apontaram a RV como uma estratégia de lazer para essa população. 
Danyane Simão Gomes,

Alanna Simão G. Saturnino, Danielle de Freitas Gonçalves,

Carlos Bandeira de M. Monteiro e Maria Georgina M. Tonello

O ensaio piloto randomizado de Prasad et al. (2018) observou que o grupo que utilizou a RV (Nintendo Wii) associada à terapia convencional (TC) produziu alterações funcionais semelhantes na função do MMSS se comparado ao grupo que realizou somente a TC. Além disto, um alto nível de satisfação foi observado em pacientes que utilizaram a RV, resultando em maior adesão ao tratamento. Desse modo, estes autores afirmam que uso do Nintendo Wii pode trazer como benefício, uma maior motivação, a qual consequentemente, leva a maior adesão ao tratamento e, maior interação, pois gera um feedback positivo por meio dos resultados, na forma de pontuação ou ao passar para níveis mais altos de dificuldade. Desta forma, podemos inferir que a maior imersão proporcionada pela RV pode gerar um maior envolvimento e interesse do indivíduo na reabilitação, tornando-o mais engajado neste processo. Assim, sugere-se novos estudos, com abordagem qualitativa, que abordem essa temática da interação e percepção dos sujeitos quanto à essa ferramenta de reabilitação.

A análise da presente revisão vai ao encontro do estudo de Prasad et al. (2018), em que nota-se que os tratamentos e a reabilitação para as pessoas com LME geralmente são duradouros, dispendiosos e exaustivos e, neste cenário, a RV se realça por promover a motivação, curiosidade, competição e interação social. Desta forma, observa-se que a RV oferece um ambiente mais envolvente dentro do contexto terapêutico, acrescentando uma dimensão de prazer, socialização e lazer, sendo estes aspectos fundamentais no contexto de vida desse grupo da população. Seguindo essa linha de pensamento, acredita-se que ao estimular a motivação e interação social, possa se promover a saúde e melhorar a qualidade de vida, já que estão relacionadas não só ao bem-estar físico, mas também mental, psicológico, emocional e social. 
Danyane Simão Gomes,

Alanna Simão G. Saturnino, Danielle de Freitas Gonçalves,

Carlos Bandeira de M. Monteiro e Maria Georgina M. Tonello

Os resultados de Dimbwadyo-Terrer et al. (2016a) corroboram com estes achados de Prasad et al. (2018), no qual investigaram os efeitos da TC combinada com um programa de RV (Toyra) na função dos MMSS de pessoas com tetraplegia completa subaguda. Esses resultados mostraram que a RV associada à TC (terapia ocupacional, exercícios funcionais e fisioterapia) produziu mudanças funcionais semelhantes no desempenho dos MMSS se comparado ao grupo que realizou somente a TC. Os autores acreditam que a duração das intervenções possa explicar essa falta de diferença entre os grupos e que a inserção de variáveis cinemáticas poderiam aumentar a fidedignidade do trabalho e reforçar a utilidade da RV para estudar e tratar distúrbios motores. Neste sentido, sugere-se novos estudos, com diferentes metodologias que desenvolvam programas de reabilitação associados à RV com durações prolongadas, além do incremento de uma maior quantidade de sessões realizadas. Observou-se também um alto nível de satisfação dos pacientes que utilizaram a RV. De acordo com os autores, os participantes do estudo expressaram o desejo de usar o Toyra em sua reabilitação diária e em casa, além disso recomendariam o uso desse sistema a outros e por isso, afirmaram que a motivação dos pacientes poderia ser um importante preditor de mudanças em longo prazo em qualidade de vida e desfechos de reabilitação. Em conformidade com esses autores, acredita-se que a oportunidade de interagir por meio de jogos eletrônicos, pode ajudar a manter a autoestima e a criar experiências favoráveis para estes indivíduos, oferecendo momentos de socialização e entretenimento.

Com o objetivo de treinar o alcance de movimento dos MMSS após a LME, Dimbwadyo-Terrer et al. (2016b) testaram o uso de uma luva combinada com um ambiente de RV, apresentando efeitos positivos. Os pacientes deste estudo apresentaram mudanças nos valores dos movimentos ativos e parâmetros funcionais no grupo 
Danyane Simão Gomes,

Alanna Simão G. Saturnino, Danielle de Freitas Gonçalves,

Carlos Bandeira de M. Monteiro e Maria Georgina M. Tonello

experimento. Diante disso, eles apostam na hipótese de que com o treinamento virtual, os pacientes podem ter transferido seu aprendizado para objetos reais diários. Entretanto, é importante destacar o pequeno tamanho da amostra do estudo-piloto em questão, sugerindo-se assim, novos estudos com amostras significativas.

Ainda analisando a função dos MMSS, Trincado-Alonso et al. (2014) observaram que dados cinemáticos registrados pelo sistema de RV Toyra se correlacionam com escalas clínicas específicas para a função dos MMSS. Estes autores apoiam o uso de tais sistemas de RV não apenas como ferramentas de reabilitação, mas também como um instrumento de avaliação objetivo do desempenho do usuário, fornecendo dados com potencial relevância clínica. Acreditam que essa informação possa complementar os escores de escalas clínicas, e assim, projetar tratamentos que incentivem as articulações ligadas a uma melhora funcional. A partir desses resultados sugerem o desenvolvimento de um sistema que poderia oferecer ao profissional/ clínico uma estimativa do escore da escala esperado para um paciente, adicionando assim, dados que poderiam facilitar e acompanhar a progressão dele.

O estudo de Khurana; Walia e Noohu (2017) foi desenhado para comparar a eficácia do treinamento baseado em jogos de RV no equilíbrio e no desempenho funcional em pessoas com paraplegia. Estes autores sugerem que as mudanças observadas nos participantes do grupo que realizou jogos de RV podem ser devido às maiores exigências de inquietação exigidas pelos jogos, se comparado ao treinamento específico. Sugerem que a extensão do desafio que os participantes experimentaram, controlando seu centro de gravidade durante a dinâmica das atividades, possa ter levado a mudanças perceptíveis nas medidas de equilíbrio. Os componentes de autocuidado 
Danyane Simão Gomes,

Alanna Simão G. Saturnino, Danielle de Freitas Gonçalves,

Carlos Bandeira de M. Monteiro e Maria Georgina M. Tonello

não foram muito afetados com o treinamento e justificam que pode ser devido ao fato de que a melhoria no equilíbrio e no desempenho não melhore o autocuidado.

Já Pozeg et al. (2017) buscaram investigar mudanças na propriedade corporal e dor neuropática crônica em pacientes com LME, por meio de ilusões corporais e estímulos multisensoriais. Eles identificaram que o grupo com LME integram-se menos prontamente a informações visuais e táteis disponíveis para experimentar a propriedade ilusória das pernas. Apesar dos efeitos analgésicos globais moderados na ilusão das pernas virtuais (IPV), observaram que apenas a estimulação visuotátil (associada à IPV) resultou numa redução quase significativa da dor neuropática. Afirmam que os resultados encontrados precisam ser interpretados com cautela, pois a amostra foi relativamente pequena e clinicamente heterogênea, no entanto, são relevantes para o desenho da reabilitação não invasiva e protocolos de controle da dor, sugerindo a importância de intervenções precoces para fortalecer a representação do corpo multissensorial na população com LME.

Shokur et al. (2016) propôs que a estimulação tátil foi capaz de induzir os pacientes a experimentarem uma ilusão proprioceptiva e, isso permitiu que deduzissem a posição das pernas do avatar virtual. Assim, estes autores acreditam que essas descobertas criam uma ligação entre a incorporação de membros e o remapeamento sensorial destes indivíduos, e o fato de readquirir o feedback tátil das pernas, recria a interação com o mundo externo.

Donati et al. (2016) exploraram o quanto um protocolo baseado na Interfacecérebro-computador (ICC) de longo prazo (12 meses) poderia ajudar os pacientes com LME a recuperarem sua capacidade de andar de forma autônoma, usando um exoesqueleto controlado pelo cérebro. Houve recuperação parcial dos pacientes, 
Danyane Simão Gomes,

Alanna Simão G. Saturnino, Danielle de Freitas Gonçalves,

Carlos Bandeira de M. Monteiro e Maria Georgina M. Tonello

manifestada por melhorias nas sensações somáticas e no controle motor voluntário, abaixo do nível da LME. Essa melhora sensório-motora também foi acompanhada por melhorias autonômicas, como a função intestinal. O padrão de recuperação sensorial documentado indicou um maior efeito mediado por pequenas fibras mielinizadas ou não-mielinizadas, do que através das grandes fibras mielinizadas que normalmente medeiam a discriminação tátil e proprioceptiva.

A partir disso, os autores sugerem que os axônios que atravessam o trato espinotalâmico são os principais mediadores da recuperação somatossensorial. Além disso, preconizam que as aplicações de ICC devem ser melhoradas e que esse novo tipo de tecnologia assistiva pode ajudar os pacientes a recuperarem a mobilidade, por meio do uso de próteses, demonstrando ser uma terapia de neuroreabilitação potencialmente nova, capaz de induzir a recuperação parcial de funções. De toda forma, é importante destacar o número limitado de participantes no estudo, sendo então recomendado novos estudos com amostras maiores que abordem a ICC e o uso de exoesqueletos e também que a quantidade de sessões realizadas seja descrita na metodologia.

A fim de examinar a eficácia do feedback de vídeo imediato em um ambiente de reabilitação para treinar usuários para o uso da cadeira de rodas, Wang et al. desenvolveram seu estudo em 2015. Não houve diferença significativa no tempo necessário para aprender e dominar as habilidades em cadeira de rodas entre os grupos experimental e controle, o que demonstrou que o feedback de vídeo imediato pode ser um método de treinamento alternativo para a habilidade em cadeira de rodas. Estes autores sugerem estudos futuros, incorporando indicações verbais e gráficas de computador com o feedback de vídeo, para aumentar a eficácia do treinamento. 
Danyane Simão Gomes,

Alanna Simão G. Saturnino, Danielle de Freitas Gonçalves,

Carlos Bandeira de M. Monteiro e Maria Georgina M. Tonello

Um fato que merece ser destacado é a quantidade de sessões realizadas, já que os autores afirmam terem sido realizadas 2 vezes por semana, até que o participante tenha dominado as habilidades. Sugere-se que a quantidade de sessões seja descrita no estudo e que seja avaliada de forma homogênea entre os participantes. Além disso, é importante mencionar o pequeno tamanho da amostra utilizado neste estudo, o que deve ser analisado com cuidado, já que pode não representar com segurança toda a população com LME.

Nunnerley et al. (2017) desenvolveram e testaram o uso da cadeira de rodas em um sistema de treinamento de RV por meio do envolvimento com os usuários e profissionais da reabilitação. Foi possível observar que esse sistema de treinamento forneceu uma perspectiva 360 graus do ambiente. A adição de um controlador de cadeira de rodas, em vez de um controle de computador pareceu contribuir para o realismo do instrutor de cadeira de rodas. Os participantes dirigiram em torno de um ambiente familiar e muitos sentiram que isso melhorou tanto a oportunidade de aprendizado físico quanto o realismo do sistema. Além disso, os participantes sugeriram usar ambientes comunitários como pano de fundo para o treinamento de cadeira de rodas para tornar essas habilidades clinicamente relevantes. O sistema de RV foi percebido como uma ferramenta terapêutica útil, mas também como uma aplicação que os pacientes poderiam usar de forma independente ou com a ajuda de familiares. Um efeito adverso encontrado foram as náuseas ao usar o sistema de treinamento, o que reduziu o tempo em que os participantes passaram no ambiente de reabilitação virtual.

Jordan e Richardson (2016) avaliaram os efeitos da caminhada virtual com a resolução das dores neuropáticas, apresentando dois estudos de caso, sendo o segundo, um estudo-piloto. De acordo com esses autores, há um esforço crescente para identificar 
Danyane Simão Gomes,

Alanna Simão G. Saturnino, Danielle de Freitas Gonçalves,

Carlos Bandeira de M. Monteiro e Maria Georgina M. Tonello

formas eficazes de tratamento para melhora destas dores, alternativas às farmacológicas tradicionais. Ao verificar a localização dessa dor nas condições de caminhada virtual e rodas virtual, os resultados do estudo de Jordan e Richardson sugeriram que as dores neuropáticas nas pessoas com LME foram reduzidas no tratamento com caminhada virtual, independentemente da localização delas. Estudos anteriores sugeriram que os modelos de estimulação ambulatória não invasivos poderiam reduzir a dor em pessoas com LME (ÖZKUL et al., 2015; MOSELEY, 2007) e reverter a reorganização cortical mal-adaptativa.

No estudo exploratório de Roosink et al. (2016), o efeito de feedback virtual interativo sobre o desempenho de imagens motoras durante a caminhada virtual em pessoas com LME foi avaliado. Encontrou-se que as tentativas de um avatar podem ser controladas de forma interativa, com maior vivacidade das imagens motoras e da velocidade, enquanto o esforço foi menor em comparação a ensaios em que apenas uma cena estática foi exibida. Os efeitos adversos foram menores e a imersão foi relatada como boa. Estes autores acreditam que vários mecanismos poderiam ser responsáveis pelos efeitos observados, incluindo mecanismos sensório-motores e mecanismos cognitivo-emocionais. Quanto à capacidade de imaginação motora avaliada, variações entre os participantes foram encontradas, o que foi justificado pela amostra heterogênea. No entanto, a maior capacidade imagética motora geral não foi associada ao melhor desempenho de imagens de marcha. Ainda neste mesmo estudo, nenhuma mudança na intensidade da dor neuropática foi observada e com isso, os autores inferem que o imediato efeito da imagética motora na intensidade da dor neuropática, seus potenciais mecanismos de trabalho e sua relação com a eficácia terapêutica em longo prazo, permanecem incertos em pessoas com LME. 
Danyane Simão Gomes,

Alanna Simão G. Saturnino, Danielle de Freitas Gonçalves,

Carlos Bandeira de M. Monteiro e Maria Georgina M. Tonello

Por fim, Eick e Richardson (2015) confirmaram a hipótese de que paradigmas baseados na caminhada virtual estão associados com o aumento da ativação cortical em áreas sensório-motoras em pessoas com LME e que o padrão geral de ativação foi bastante diferente dos participantes saudáveis. Desta forma, sugerem que as modalidades de tratamento baseadas em paradigmas de estímulos sensoriais (por exemplo, caminhada virtual) possam funcionar pela segmentação organizada do córtex somatossensorial que ocorre após a LME. No entanto, verificaram que participantes fisicamente capazes apresentaram maior imersão durante a simulação visual adaptada e os indivíduos foram mais capazes de se imaginarem como os atores, em comparação com pessoas com LME.

A partir da análise desta revisão, foi possível observar que houve limitação do tamanho das amostras na grande maioria dos artigos analisados e, além disso, alguns destes estudos foram estudos-piloto. Sugere-se, assim, que sejam realizados ensaios clínicos randomizados, com amostras maiores, além de uma maior quantidade e duração das sessões de RV. Destaca-se a necessidade de estudos que envolvam a abordagem quali-quantitativa da análise da eficácia e da percepção dos indivíduos quanto a este tipo de intervenção, evidenciando a motivação, o engajamento e o interesse deles quanto à prática da RV como um instrumento de reabilitação ou até mesmo, para ser utilizada em seus momentos de lazer e socialização.

Desta maneira, acredita-se que a RV possa ser utilizada como uma relevante ferramenta de reabilitação e lazer de pessoas que estão com restrições de locomoção, de transporte ou pessoas que fazem uso de próteses e/ou órteses, como no caso de pessoas com LME. 
Danyane Simão Gomes,

Alanna Simão G. Saturnino, Danielle de Freitas Gonçalves,

Carlos Bandeira de M. Monteiro e Maria Georgina M. Tonello

\section{Considerações Finais}

De acordo com os resultados apresentados podemos inferir que a maior imersão proporcionada pela RV pode gerar um maior envolvimento e interesse do indivíduo na reabilitação, tornando-o mais engajado neste processo. A RV se realça por promover a motivação, curiosidade, competição e interação social. Desta forma, observa-se que a RV oferece um ambiente mais envolvente dentro do contexto terapêutico, acrescentando uma dimensão de prazer, socialização e lazer, sendo estes aspectos fundamentais no contexto de vida dessa população.

Foi possível identificar também, que a RV é bastante empregada na reabilitação de pessoas com LME, demonstrando efeitos positivos para estimular a melhora da função dos MMSS, treinar indivíduos para o uso de cadeiras de rodas; diminuir o nível de dor neuropática; auxiliar na adaptação de exoesqueletos/ neuropróteses, recuperando a experiência perceptiva dos membros inferiores. Diante da análise dos estudos em questão, acredita-se que vários mecanismos podem ser responsáveis pelos efeitos observados, incluindo mecanismos sensório-motores e mecanismos cognitivo-emocionais. No entanto, os resultados apontam que a RV não substitui a reabilitação convencional, mas adiciona uma dimensão de prazer, oferecendo também uma oportunidade de lazer, o que pode fazer conexão direta com a promoção da saúde e a qualidade de vida de pessoas com lesão da medula espinal.

\section{REFERÊNCIAS}

AHUJA, C.S. et al. Traumatic spinal cord injury. Nature Reviews Disease primers, v.3, p.17018, 2017.

DIMBWADYO-TERRER, I. et al. Effectiveness of the virtual reality system Toyra on upper limb function in people with tetraplegia: a pilot randomized clinical trial. BioMed

Research International, v. 2016, 2016 (a). 
Danyane Simão Gomes,

Alanna Simão G. Saturnino, Danielle de Freitas Gonçalves,

Carlos Bandeira de M. Monteiro e Maria Georgina M. Tonello

DIMBWADYO-TERRER, I. et al. Upper limb rehabilitation after spinal cord injury: a treatment based on a data glove and an immersive virtual reality environment. Disability and Rehabilitation: Assistive Technology, v.11, n.6, p.462467, 2016 (b).

DONATI, A.R.C. et al. Long-term training with a brain-machine interface-based gait protocol induces partial neurological recovery in paraplegic patients. Scientific reports, v. 6, p.30383, 2016.

EICK, J.; RICHARDSON, E.J. Cortical activation during visual illusory walking in persons with spinal cord injury: A pilot study. Archives of physical medicine and rehabilitation, v. 96, n. 4, p. 750-753, 2015.

JORDAN, M.; RICHARDSON, E.J. Effects of Virtual Walking Treatment on Spinal Cord Injury-Related Neuropathic Pain: Pilot Results and Trends Related to Location of Pain and at-level Neuronal Hypersensitivity. American journal of physical medicine \& rehabilitation, v. 95, n. 5, p. 390-396, 2016.

KHURANA, M.; WALIA, S.; NOOHU, M.M. Study on the effectiveness of virtual reality game-based training on balance and functional performance in individuals with paraplegia. Topics in spinal cord injury rehabilitation, v.23, n.3, p.263-270, 2017.

KUMRU, H. et al. The effects of transcranial direct current stimulation with visual illusion in neuropathic pain due to spinal cord injury: an evoked potentials and quantitative thermal testing study. European Journal of Pain, v. 17, n. 1, p. 55-66, 2013.

MOSELEY, G. Lorimer. Using visual illusion to reduce at-level neuropathic pain in paraplegia. Pain, v.130, n.3, p.294-298, 2007.

NUNNERLEY, J. et al. Training wheelchair navigation in immersive virtual environments for patients with spinal cord injury-end-user input to design an effective system. Disability and Rehabilitation: Assistive Technology, v.12, n.4, p.417-423, 2017.

ÖZKUL, Ç. et al. Effects of visual illusion and transcutaneous electrical nerve stimulation on neuropathic pain in patients with spinal cord injury: A randomised controlled cross-over trial. Journal of back and musculoskeletal rehabilitation, v.28, n.4, p.709-719, 2015.

POZEG, P. et al. Virtual reality improves embodiment and neuropathic pain caused by spinal cord injury. Neurology, v.89, n.18, p.1894-1903, 2017.

PRASAD, S. et al. Efficacy of Virtual Reality in Upper Limb Rehabilitation in Patients with Spinal Cord Injury: A Pilot Randomized Controlled Trial. Asian spine journal, v.12, n.5, p.927, 2018.

ROOSINK, M. et al. Interactive virtual feedback improves gait motor imagery after spinal cord injury: an exploratory study. Restorative neurology and neuroscience, $v$. 34 , n. 2, p. 227-235, 2016. 
Danyane Simão Gomes,

Alanna Simão G. Saturnino, Danielle de Freitas Gonçalves,

Carlos Bandeira de M. Monteiro e Maria Georgina M. Tonello

SERRA, M.V.G.B.; FAVA, M.C.; TONELLO, M.G.M. Realidade virtual para pessoas com deficiência: o uso do video game como prática de lazer. Licere (Online), v. 21, n. 4, p. 529-548, 2018. Disponível em: https://periodicos.ufmg.br/index.php/licere/article/view/1952. Acesso em: 13 abr. 2019.

SHOKUR, S. et al. Assimilation of virtual legs and perception of floor texture by complete paraplegic patients receiving artificial tactile feedback. Scientific reports, v.6, p.32293, 2016.

TRINCADO-ALONSO, F. et al. Kinematic metrics based on the virtual reality system toyra as an assessment of the upper limb rehabilitation in people with spinal cord injury. BioMed research international, v.2014, 2014.

WALL, T. et al. The effects of the Nintendo ${ }^{\mathrm{TM}}$ Wii Fit on gait, balance, and quality of life in individuals with incomplete spinal cord injury. The journal of spinal cord medicine, v.38, n.6, p.777-783, 2015.

WANG, Y.T. et al. Immediate video feedback on ramp, wheelie, and curb wheelchair skill training for persons with spinal cord injury. Journal of Rehabilitation Research \& Development, v.52, n.4, p.421-431, 2015.

ZIMMERLI, L. et al. Increasing patient engagement during virtual reality-based motor rehabilitation. Archives of physical medicine and rehabilitation, v.94, n.9, p.17371746, 2013.

\section{Endereço dos Autores:}

Danyane Simão Gomes

Avenida Padre Almir Neves de Medeiros, 430 apto 401 - Bairro Sobradinho

Patos de Minas - MG - 38.701-118

Endereço Eletrônico: danyanesg@hotmail.com

Alanna Simão Gomes Saturnino

Avenida Padre Almir Neves de Medeiros, 430 apto 401 - Bairro Sobradinho

Patos de Minas - MG - 38.701-118

Endereço Eletrônico: Nana417@hotmail.com

Danielle de Freitas Gonçalves

Rua Paulo Augusto Fonseca, 926 - Bairro Ipanema II

Patos de Minas - MG - 38.706-496

Endereço Eletrônico: daniellegoncalvesmg@gmail.com

Carlos Bandeira de Mello Monteiro

Rua Arlindo Béttio, 1000 - Bairro Ermelino Matarazzo

São Paulo - SP - 03.828-000

Endereço Eletrônico: carlosmonteiro@usp.br 
Danyane Simão Gomes,

Alanna Simão G. Saturnino, Danielle de Freitas Gonçalves,

Carlos Bandeira de M. Monteiro e Maria Georgina M. Tonello

Maria Georgina Marques Tonello

Avenida Dr. Armando de Salles Oliveira, 201 - Bairro Parque Universitário

Franca - SP - 14.404-600

Endereço Eletrônico: gina@ginatonello.com.br 